\title{
Retraction Note to: A Study on the Percolation Threshold of Polyethylene Matrix Composites Filled Carbon Powder
}

\section{Soon-Gi Shin ${ }^{1}$}

Published online: 23 February 2018

(c) The Korean Institute of Metals and Materials 2018

The Editor-in-Chief and Editorial Board of Electronic Materials Letters have retracted this article [1] because its contents have been previously published by Nakamura et al. [2]. The contents of this article are therefore redundant. Author Soon-Gi Shin has not responded to correspondence from the editor about this retraction.

The original article can be found online at https://doi.org/10.3365/ eml.2010.06.065.

Soon-Gi Shin

ssg@ kangwon.ac.kr

1 Department of Advanced Materials Engineering, College of Samcheok, Kangwon National University, Samcheok-si, Gangwon 245-711, Korea

\section{References}

1. Shin, S.G.: Electron. Mater. Lett. 6, 65 (2010). https://doi. org/10.3365/eml.2010.06.065

2. Nakamura, S., Saito, K., Sawa, G., Kitagawa, K.: Jpn. J. Appl. Phys. 36, 5163 (1997). https://doi.org/10.1143/JJAP.36.5163 